\title{
RASGOS FONÉTICOS DEL CARÁCTER INTERFERENCIAL EN EL CASTELLANO DE UNA COMUNIDAD BILINGÜE
}

\author{
María del Carmen Serrano Vázquez \\ (Universidad de Valladolid)
}

\begin{abstract}
RESUMEN
Castilian of the Balearic Isles can be analyzed under a point of view of languages in contact. We appreciate this as in the sphere of the individual that uses both languages for communicative purposes, as in the sphere of a society where, in a diglosic way, exists the tendency to employ one tongue or another, taking into account sociolinguistics parameters (home, vicinty, work, administration, mass-media) and where Castilian language is still associated with the sociopolitical and cultural groups of prestige.

From the real proves of usage taken along the fieldwork that we have carried out with the scholastic community of the Public School «Es Puig» from Sóller (Balearic-Isles) we have obtained series of phonetics features which show us a remarkable influence of Catalan language patterns over Castilian, in this linguistic community.

This linguistic features move away from the standard of Castilian in other geographical areas; they are provoked by the environmental bilingualism which exists in the Balearic Community and present a great vitality stimulated by the progressive social normalization of Catalan language, which reach the acceptation of many of them by the non-bilingual Balearic Community.
\end{abstract}

\section{Marco referencial}

El castellano de las Islas Baleares se puede analizar desde una perspectiva de lenguas en contacto. La situación lingüística actual de las Islas Baleares presenta un esquema claro de bilingüismo social; es decir, se trata de «una comunidad en cuyo interior funcionan y son utilizadas dos lenguas distintas» (Rojo, 1981:270)․

1 Para una crítica del concepto de bilingüismo social, vẻanse Aracil (1973) y Mollà y Palanca (1987), entre otros. 
En este bilingüismo la igualdad entre las dos lenguas en presencia, castellano y catalán, no existe. Su coexistencia está marcada por un desequilibrio a favor del castellano, presentando por tanto un carácter diglósico. En su acepción más corriente, diglosia, término difundido por Ferguson (1959: 325-340)², significa que «en toda situación de coexistencia de dos lenguas se produce un desequilibrio en favor de una de ellas, lo que permite calificar a una de ellas de lengua fuerte y a la otra de lengua débil» (Siguan y Mackey, 1986: 45).

Esta existencia implica también en la actualidad una tensión y una dinámica en la que una de las lenguas, el catalán, amenaza con desplazar a la otra, el castellano, ya que las dos comienzan a usarse, indistintamente, para varias funciones (en la educación, en la administración, por ejemplo) reservadas antes a una de las lenguas, el castellano. Estaríamos, siguiendo la tipología utilizada por Lewis (1972), en una fase de bilingüismo dinámico. Por otro lado, sin embargo, el mayor uso oficial y público del catalán — consecuencia inmediata de la campaña en defensa de la lengua catalana- coincide con su retroceso en los usos personales y sociales, destacando entre éstos la espectacular castellanización de los ambientes juveniles (Bibiloni, 1988).

El bilingüismo social ${ }^{3}$ genera en la comunidad balear un determinado grado de bilingüismo individual ${ }^{4}$, hablantes que han logrado un dominio aceptable de los dos sistemas lingüísticos, que son capaces de utilizar dos lenguas con la misma o parecida facilidad (Siguan, 1976: 28-29): «El bilingüismo como forma de comportamiento de un individuo se define como la capacidad para utilizar indiferentemente, con la misma competencia y en las mismas situaciones dos lenguas distintas». Este bilingüismo perfecto se constituye en la medida del bilingüismo, y así consideraremos que un individuo es tanto más bilingüe cuanto más se acerque a esta igualdad, a este modelo ideal ${ }^{5}$.

2 Una formulación más extensa del concepto de diglosia se la debemos a Fishman (1967: 29 38), que diferenció cuatro modalidades de coexistencia linguística. Para otras modificaciones aportadas al concepto de diglosia se puede acudir al trabajo de sintesis de Daoust y Maurais (1987: 5-46), y a la revisión del término que ofrece Tabouret-Keller (1982: 17-43).

3 Para una tipología del bilingüismo social se puede acudir a Baetens (1986: 5), Lewis (1972), Sánchez Carrión (1981: 51).

4 El grado de exigencia que adopta un autor para denominar a un hablante como bilingüe es variable. Si Haugen (1953:7) adopta el máximo de flexibilidad al considerar que el bilingüismo empieza cuando el hablante de una lengua puede producir enunciados completamente significativos en la otra, autores como Halliday, Mckintosh y Strevens (1968: 139-169) establecen la exigencia máxima y hablan de "ambilinguiismo» para referirse a ese individuo que es capaz de funcionar igualmente bien en cualquiera de las lenguas, en todos los dominios de su actividad, y sin trazas de una de ellas en.el uso de la otra, mientras que otros estudiosos del fenómeno como Siguan (1976: 7981) proponen una cuantificación del bilingüismo introduciendo una gradación en la competencia lingüística de las dos lenguas, que nos daría una imagen más matizada de la realidad.

5. Rotaetxe (1988: 55-56) nos ofrece una tipología del bilingüismo como hecho individual diferenciando 1) el grado de dominio de cada uno de los dos sistemas linguíísticos y 2) la utilización hecha de tales sistemas en la conducta social. 
El bilingüismo ambiental que existe en la comunidad balear provoca hechos de interferencia en el discurso de los hablantes, individuos que usan las dos lenguas y que son el punto de contacto (Weinreich, 1974: 17): «Dos o más lenguas están en contacto si son usadas alternativamente por las mismas personas. Los individuos que usan las lenguas son, por lo tanto, el punto de contacto».

El término de interferencia como tal fue introducido por Sandfeld (1936), pero fue Weinreich (1953) quien ofreció el estudio más exhaustivo de las distintas formas y causas de interferencia. Éste la definió como «casos de desviación con respecto a las normas de cualquiera de las dos lenguas que ocurren en el habla de los individuos bilingües como resultado de su familiaridad con más de una lengua, es decir, como resultado de contacto». (Weinreich, 1974: 17-18).

Haugen (1973: 551), sin embargo, restringió el término interferencia a la «superposición simultánea de dos normas lingüísticas al mismo elemento», como resultado de la incapacidad o indiferencia de los individuos bilingües a mantener los dos códigos enteramente separados. Así pues, cuando la desviación acaba siendo adoptada por la comunidad deja de ser interferencia y puede decirse que ha sido integrada. En ese mismo sentido, Mackey (1976) distingue entre interferencia e integración. La primera se produce en el habla y la segunda en la lengua; la interferencia es individual y extrasistemática y la integración, colectiva y sistemática. Un elemento es interferencia en tanto no se integre en la lengua afectada; cuando lo hace, se adapta fonológica, gramatical y léxicamente al sistema, integrándose en él.

Este problema ha ocupado a un buen número de especialistas en el terreno del bilingüismo. El lingüista catalán Payrató (1985: 58) discute críticamente la dicotomía de Mackey y propone que se utilice el término interferencia en un sentido amplio como denominación global, de conjunto, de un grupo natural de casos de cambio lingüístico: «una interferència, en sentit ampli, és un canvi lingüístic (= una innovació, una pèrdua, una substitució) que té lloc en una llengua $A$ (o registre), i que és motivat directament per la influència d'una llengua B (o d'un altre registre de la mateixa llengua, si així s'especifica)».

Payrató presenta una clasificación de los fenómenos de interferencia en la que tiene presente un desarrollo progresivo de los mismos. A su juicio, son cuatro los estadios que cabría analizar en el estudio de la interferencia dentro de una comunidad de habla: 1) Elementos de procedencia foránea adaptados a lo largo de la historia de una lengua: germanismos, arabismos .... 2) Elementos procedentes de una segunda lengua aceptados sólo recientemente por la normativa actual: el adjetivo catalán maco —interferencia del castellano majoincluido ya en los diccionarios de catalán. 3) Elementos también de procedencia externa al sistema y de uso generalizado entre los hablantes, pero no aceptados por la normativa de la comunidad. El punto de referencia puede estar en el uso 
de estos elementos por parte de los hablantes monolingües: algunas perífrasis del catalán contemporáneo como tenir que..., donar-se compte ..., interferencias del castellano que están desplazando a las genuinas haver de..., adonar-se. 4) Rasgos o elementos foráneos (que pertenecen a una lengua $A$ ), que utiliza un hablante bilinguie cuando se expresa en una lengua $B$. No se dan en los monolingües, ni la normativa los acepta.

Estos fenómenos forman parte de un proceso unitario, que se produce en el orden inverso al expuesto: desde el acto singular en el habla de los bilingües hasta la interferencia como un hecho histórico. El concepto de interferencia en sentido estricto es el que se da en el caso 4 ): «trets o elements que corresponen a un altre sistema i que són utilitzats per parlants bilingües». (Payrató, 1985: 59).

Para Payrató (1985: 60) el uso más sensato del término sería considerar los cuatro casos como noción genérica, que puede ser restringida al habla de los bilingües (caso $4^{\circ}$ ) cuando sea preciso. Según el tipo de trabajo se justificará un uso estricto de la noción de interferencia, restringida al habla de los bilinguies o un uso amplio donde la interferencia se conciba como un proceso unitario. Cuando nosotros analicemos el habla de los escolares del colegio Es Puig de Sóller, objetivo de nuestra investigación, utilizaremos el término de interferencia en su uso restringido.

Para la sociolingüística actual la variedad de usos que un grupo de hablantes tiene la posibilidad de realizar utilizando un único sistema lingüístico, las divergencias de estructuras que pueden estar ligadas a esta diversidad de usos, así como también los hechos de interferencia en el discurso de los hablantes, producidos por la existencia de más de un sistema lingüístico en la comunidad, es lo que define los conceptos de variación -intralingüística o interlingüísticay de variedades lingüísticas. De este modo «la interferencia lingüística no deberá reducirse a simples desviaciones o variaciones de la norma, sino a variantes o variables relativas a ciertos subgrupos y circunstancias, regidas por las normas sociolingüísticas de la comunidad de habla que deben ser descubiertas y analizadas». (Etxebarría, 1986: 70). Como fenómenos sistemáticos que ocurren en el habla de las comunidades bilingües Romaine (1988: 286), por su parte, ha señalado que fenómenos como la interferencia o el code-switching no pueden ser vistos como desviaciones de una organización gramatical básica, sino que al contrario, ellos mismos constituyen la propia gramática de esas comunidades.

La causa lingüística de la interferencia es la dicotomía provocada por el hecho de que las lenguas se diferencian y se asemejan a la vez. El mecanismo productor es la identificación interlingüística de unidades: el hablante identifica los signos de su sistema $(\mathrm{O})$ (de significado o de significante) con los signos de la lengua productora de interferencias $(F)$, en razón de similitudes de forma esenciales o parciales. A partir de ahí, el bilingüe aplica a los elementos identificados las normas de F que pueden o no coincidir con las de O. Por ello, 
es normal que el análisis de las interferencias siga los niveles del análisis descriptivo (fónico, gramatical, léxico), en las lenguas $F$ y $O$.

Aparte de analizar el inicio del proceso, estudiar las interferencias obliga a referirnos a todo el proceso (difusión, persistencia, desaparición) y a las diversas causas que en él intervienen, a los factores estructurales (los que conciernen a las lenguas como sistemas) y a los no estructurales (los extralingüísticos). Unos y otros actúan juntos y el resultado de su síntesis es el que lleva en última instancia al desarrollo de la interferencia.

Entre los factores estructurales cabe señalar 1) la economía (Weinreich, 1953/1974: 2-11; Haugen, 1953: 380-8; Van Overbecke, 1976: 123): supresión de los elementos más específicos de cada lengua y generalización de los elementos comunes o más próximos y adaptables, acompañada de intercambios y modificaciones fonético-fonológicas, semánticas y sintácticas de todo tipo;2) la frecuencia de las unidades en el discurso; 3) la inteligibilidad, la posibililidad de hacerse entender o comunicabilidad; el peligro de confusión o mala interpretación; 4) la estabilidad (Haugen, 1953: 405-8) y cohesión de un sistema o subsistema, y el grado de integración de cada elemento lingüístico (Appel y Muysken, 1987: 12).

Entre los no estructurales Weinreich (1974: 21-22) señala que algunos son inherentes a la relación de la persona bilingüe con las lenguas que pone en contacto: a) la facilidad de expresión verbal que tiene el hablante en general y su habilidad para mantener separadas las dos lenguas; b) la habilidad relativa en cada lengua; c) la especialización en el uso de cada lengua por temas e interlocutores; d) la manera de aprender cada lengua; e) las actitudes hacia cada lengua, ya sean éstas idiosincráticas o estereotipadas. Entre los factores no estructurales característicos del grupo considerado como un todo Weinreich (1974: 22-23) señala como relevantes: f) el tamaño del grupo bilingüe y su homogeneidad o diferenciación sociocultural; 1a división en subgrupos que usan una u otra lengua como lengua materna, los datos demográficos; las relaciones sociales y políticas entre esos subgrupos; g) el predominio de individuos bilingües con ciertas características de comportamiento lingüístico dentro de los distintos subgrupos; h) las actitudes estereotipadas hacia cada lengua (el «prestigio»), la condición de indígenas o inmigrantes de las lenguas en cuestión; i) las actitudes hacia la cultura de cada comunidad lingüística; j) las actitudes hacia el bilingüismo en sí; k) la tolerancia o la intolerancia con respecto a la mezcla de lenguas y a los usos incorrectos en cada lengua; 1) las relaciones entre el grupo bilingüe y las comunidades unilingües.

Tanto los factores estructurales como los no estructurales pueden favorecer o inhibir el nacimiento de la interferencia al mismo tiempo que acelerar o aminorar la velocidad del proceso: «los fenómenos de interferencia son considerados como resultantes de dos fuerzas opuestas: los estímulos de las 
interferencias y la resistencia a las interferencias». (Weinreich, 1974: 139). Tanto los unos como los otros pueden ser estructurales o no estructurales.

Todavía hoy se mantienen numerosas discrepancias entre los investigadores sobre muchos aspectos relacionados con la interferencia lingüística; entre ellos, la propia delimitación del fenómeno con respecto a otros.

En ocasiones puede resultar difícil decidir acerca de si un determinado fenómeno es debido a la influencia de otro sistema lingüístico o a cambios internos en la propia lengua. El tratamiento de estos casos ha llevado a algunos lingüistas a la creación del término convergencia para diferenciarlo de la interferencia (Payrató, 1985).

Para calificar los fenómenos linguísticos resultantes del contacto de lenguas se utilizan también términos como extranjerismos, préstamos, calcos, codeswitching.

Atendiendo al grado de incorporación al sistema de una lengua, Seco (1977: 197) distingue entre voces extranjeras adaptadas totalmente a la lengua, préstamos, y voces que todavía se sienten enquistadas en el idioma, extranjerismos. En este sentido, en el nivel léxico la noción de interferencia (en sentido estricto) cubriría el mismo terreno que los extranjerismos no adaptados (Payrató, 1985: 59).

Un tipo especial de préstamo es el calco, unidad léxica que presenta un significante en la lengua objeto, pero que tiene un significado calcado del de un signo de la lengua fuerte (castellano rascacielos < inglés sky-scraper), que puede extenderse a la sintaxis, a las locuciones.

La calificación de error para referirse al fenómeno de la interferencia tampoco es adecuada, por el carácter sistemático que caracteriza a ésta. El error es una forma marcada, un caso de desviación respecto de una actuación esperada, no marcada. El error (Payrató, 1985: 62): «té sentit comparat amb la forma correcta, i suposa una irregularitat respecte d'una determinada norma (conducta normal)». La interferencia no es un error debido al azar o a las limitaciones propias de la actuación. La interferencia tiene un carácter sistemático en relación a una segunda lengua, la cual nos aclara, nos explica, al menos en buena parte, el motivo de las desviaciones (Payrató, 1985: 61).

La diferencia entre las nociones de interferencia e integración y la de codeswitching se la debemos a Haugen quien en 1956 estableció que mientras aquellas podían acercar los dos códigos, el code-switching los mantenía diferenciados (Rotaetxe, 1988: 106). Por intercambio de códigos entendemos en un sentido amplio el cambio / alternancia de lengua o de variedad lingüística efectuado por el mismo hablante durante un hecho de habla. (Gardner - Chloros, 1983: 21).

Si la interferencia tiende a la adaptación, a integrarse en el sistema a diferencia del code-switching, no siempre es sencillo separar los dos fenómenos. Cuando el cambio de código afecta a una sola palabra, las distancias se 
acortan, con lo que podríamos considerar este caso como una interferencia en una primera fase de adaptación. (Payrató, 1985: 71).

Desde la perspectiva del estructuralismo la interferencia provoca en el sistema receptor una reestructuración de los elementos y de las relaciones establecidas entre ellos. La causa de la reestructuración se puede encontrar en uno de los seis procesos o fenómenos de interferencia siguientes, formulados los cuatro primeros por Weinreich (1974: 50-52) para el nivel fónico, y los otros dos por Payrató (1985: 79):

1) Subestimación de una distinción: los hablantes de una lengua dejan de realizar una oposición funcional existente en la misma, por influencia de otra lengua en la que tal oposición no existe.

2) Sobrestimación de una distinción: al contrario que en el caso anterior, la lengua receptora realiza una oposición estructural que no existe en la norma, como consecuencia del influjo de otra lengua en la que sí existe tal oposición.

3) Reinterpretación de una distinción: Es el caso más problemático porque implica un cambio de la relación entre los elementos de la lengua $\mathrm{L}_{2}$, no de los mismos elementos, por la influencia de la $\mathrm{L}_{1}$.

4) Sustitución de un elemento (o de una relación de elementos) de una lengua por otro de la lengua contraria. Es la categoría más general, pero exige normalmente una estrecha semejanza entre los elementos implicados.

5) Importación o simple transferencia de un elemento de la $L_{1}$ a la $L_{2}$, en la que este elemento extraño no sustituye a otro propio.

6) Pérdida de un elemento de la $\mathrm{L}_{2}$, que se deja de utilizar por influencia de la $L_{1}$, que no posee tal elemento.

\section{Metodología}

Partiendo de la hipótesis de que la influencia del catalán sobre el castellano hablado por la comunidad balear es notable, nos propusimos como objetivo de la presente investigación descubrir las peculiaridades propias del castellano hablado por una población escolar, como fruto del contacto de lenguas.

Al considerar los factores lingüísticos el foco central del estudio hemos escogido una muestra homogénea en cuanto a edad y nivel educativo. Los hablantes seleccionados constituyen un grupo social compacto porque tienen un dominio común de trabajo, el Colegio Público Es Puig de Sóller (Baleares). La muestra representativa incluye 97 sujetos (54 varones y 43 mujeres), con una edad que oscila entre los once y los quince años, alumnos de $6^{\circ}$ y $8^{\circ}$ del Ciclo Superior de EGB en el curso escolar de 1989-1990.

Una vez que teníamos diseñada la investigación y realizada la selección de la muestra de hablantes, el paso siguiente lo constituía la recolección de los datos lingüísticos en la comunidad de habla elegida, para su posterior análisis e interpretación. 


\section{II.A. Recogida de datos}

Establecimos unos instrumentos de recogida de información: a) Cuestionario socioeconómico, linguístico y actitudinal ${ }^{6}$; b) pruebas lingüísticas.

II.A.1. Cuestionario: En el cuestionario se abordaban varios temas: a) las características del sujeto; b) el conocimiento que los miembros de la población encuestada tiene de cada una de las lenguas utilizadas; c) la forma y la frecuencia de uso de las lenguas en distintas situaciones; d) las actitudes de los escolares respecto a las lenguas.

La proporción lingüística originaria: 51 (catalán) / 37 (castellano), que se basa en la lengua materna -siguiendo un criterio de anterioridad cronológica- es el punto de partida para caracterizar a una población que hay que considerar bilingüe (un $84,5 \%$ emplea habitualmente las dos lenguas y un $13,3 \%$ se considera capaz de utilizar las dos lenguas con la misma competencia); bilingüismo que presenta un leve desequilibrio en contra del catalán, según se aprecia en los indices de diglosia que atienden a distintos grados de conocimiento de las dos lenguas, y que oscilan entre el 1,26 y el 1,38 a favor de la lengua castellana.

La relación entre los indicadores del bilingüismo y las características sociales no es significativa. El grado de instrucción y el nivel social es tan similar en los distintos grupos lingüísticos que no nos permiten inferir resultados diferenciadores.

Además de los datos que hacen referencia al conocimiento de las dos lenguas por parte de la población hemos detallado cómo en el comportamiento de un individuo el uso lingüístico puede distribuirse entre las dos lenguas, según distintas situaciones y en relación con qué personas.

En el análisis de las relaciones familiares ha sido interesante conocer que el comportamiento de los padres entre sí es monolingüe, ya que sólo un 7,2\% practica un uso bilingüe. Constatamos igualmente la asimilación a la sociedad lingüística dominante de un $32,4 \%$ de padres que tienen su origen en zonas castellanoparlantes, abandonando su lengua materna como lengua familiar y aceptando la lengua autóctona. Este índice nos sirve para medir la fuerza expansiva del catalán.

Respecto a los hijos tenemos que señalar que sólo un $6,1 \%$ de los niños encuestados es bilingüe en su comportamiento lingüístico familiar. Destacamos un $24,3 \%$ de escolares que perteneciendo al grupo castellano opta por el catalán en sus relaciones familiares, en un intento de integración en la sociedad lingüís-

- Necesario para el análisis del comportamiento lingüístico de la población encuestada (Serrano, 1990). 
tica autóctona, bajando pues el índice de asimilación alcanzado en la generación ascendente.

En las relaciones sociales estos porcentajes se disparan significativamente a un $50,5 \%$ y a un $56,7 \%$ en el trato amistoso y en el escolar respectivamente. El uso de una segunda lengua viene dado por la necesidad de entrar en contacto con otros miembros de la comunidad. Cuando no se opta por este bilingüismo, la lengua castellana es la que va a dominar en el uso monolingüe por parte de los escolares. Por otra parte, es en el grupo lingüístico que tiene el castellano como lengua materna donde ésta se mantiene en el uso social de un modo exclusivo. Parece, pues, que es el grupo lingüístico castellano el que se siente menos abocado a adaptarse a la otra lengua en presencia.

El uso de las lenguas en la lectura arroja un $62,8 \%$ de bilingüismo, destacando que en el ámbito del catalán ningún miembro tenga hábitos monolingües en la lectura, frente a ese 48,6\% del grupo castellano que sólo lee en su lengua materna. En la audición los escolares optan en primer lugar por los canales de televisión de ámbito estatal TVE 1, con una programación en la que se alternaban las dos lenguas (cuando hicimos la encuesta se recibía la Segunda Cadena de Televisión Española correspondiente al circuito catalán); sólo un 1,3 $\%$ de ellos prefiere en primer lugar el canal TV 3, que emite exclusivamente en lengua catalana. Este dato es muy importante para ilustrar la dinámica de la relación entre las dos lenguas, puesto que la televisión es hoy un medio decisivo, ya que su ámbito de difusión no sólo es más amplio que el de la escuela sino que también alcanza a toda la población al mismo tiempo y cualquiera que sea su edad, convirtiéndose junto a la escuela y el periódico en los instrumentos principales para una política lingüística. Además de ese 71,1 $\%$ de bilingüismo que se da en la escritura, sobresale este dato: en ninguno de los grupos lingüísticos tratados se da el uso exclusivo del catalán en la expresión escrita, no ocurriendo lo mismo en el caso del castellano. Así, un $25,7 \%$ se expresa por escrito solamente en castellano.

En la actitud que los escolares muestran hacia las dos lenguas en presencia analizamos la valoración subjetiva, deduciendo que un 71,1\% de escolares se siente más identificado emocionalmente con su lengua materna, y la valoración objetiva donde, independientemente de las respuestas combinadas, el orden de preferencia de las lenguas según la posibilidad que ofrecen para progresar en sociedad es el siguiente: castellano $(34 \%)$, inglés $(24,7 \%)$ y catalán $(14,4 \%)$, reflejando de esta manera una diferencia de poder y prestigio entre las lenguas.

En definitiva, la población escolar encuestada es bilingüe, ya que utiliza normalmente dos lenguas distintas como medio de comunicación. Sin embargo, las dos lenguas en presencia no tienen exactamente la misma difusión ni el mismo estatus, por lo que habría que definir la situación como de bilingüismo con diglosia. 
La comparación efectuada entre los comportamientos lingüísticos de los distintos grupos, caracterizados según su lengua materna, nos ilustra sobre la relación activa entre las dos lenguas tratadas, catalán y castellano, y sobre sus perspectivas futuras.

\section{II.A.2. Pruebas lingüísticas}

\section{II.A.2.1. Pruebas orales}

Conversación de grupo. Grabamos conversaciones de grupo donde empleamos la técnica de participante-observador, a fin de hacer grabaciones de interacciones lingüísticas en situaciones creadas espontáneamente donde el habla usada es natural y espontánea y se presupone que, tanto en cuanto a los contenidos temáticos como en cuanto a las formas lingüísticas, se corresponde con el habla que usan los escolares cuando el observador no está presente.

Estas grabaciones se hicieron en un recreo y en una excursión al campo. Las conversaciones grabadas eran libres, puesto que no se ejercía ninguna clase de control ni sobre los temas ni sobre la participación de los hablantes.

Prácticas interactivas. Grabamos una serie de ejercicios realizados en grupos o por parejas de alumnos, que se hicieron en el aula: situaciones imaginarias de encuentro; describir el propio carácter, el de un compañero; entrevistas; viñetas en las que se ilustran una serie de escenas; juegos.

Conversación individual. En tercer lugar nos decidimos por grabar una conversación individual, que había sido concertada con anterioridad con el profesor. Los temas de la conversación se modificaban según fueran las respuestas de los alumnos, según su madurez personal, sus vivencias, intereses, preferencias; según el ritmo que lográramos en nuestra charla particular. En general, desarrollamos los siguientes tópicos: La situación del informante, las costumbres, los gustos, los deportes, las diversiones, las fiestas, las vacaciones.

Después de que el alumno hubiera contestado a algunas preguntas sobre su vida cotidiana, de modo que venciera su posible timidez y olvidara que estaba siendo grabado, realizamos una prueba fonética.

Prueba fonética. Habíamos preparado una selección de frases que el alumno leía mientras grabábamos, del tipo de: La vaca tuvo un percance cuando comía hierba con los animales. El saxofonista estuvo exento del examen.

Se trataba de un ejercicio más formal, ya que el niño tendía en mayor medida que en la conversación individual a la autoobservación y a la autocorrección, restando así espontaneidad a su habla.

Prácticas a partir de la imagen. En estas actividades el alumno goza de una mayor libertad y alcanza también mayor creatividad comunicativa, movilizando de forma espontánea sus recursos lingüísticos. 


\section{II.A.2.2. Pruebas escritas}

Cuadernos de deberes. Dispusimos de los cuadernos de deberes realizados en todo el curso correspondientes a la asignatura de Lengua Castellana. En ellos nos encontramos con muchas variantes de textos: Ejercicios de gramática (dictados, comentarios de texto, controles de la materia, exámenes...); resúmenes; anuncios publicitarios inventados; descripciones; mi biografía; recetas de cocina; cuentos inventados; redacciones sobre temas sugeridos o libres.

Estructuras diferenciadas. Otro medio de determinar la existencia de interferencias fue seleccionar una serie de estructuras diferenciadas en castcllano y en catalán del libro de Jordana (1968) y elaborar una serie de textos con huecos en blanco, que el alumno tenía que rellenar con las expresiones deseadas.

Después de la recogida de datos mediante una serie de pruebas orales y escritas hemos obtenido una serie de rasgos o elementos que pertenecen a la lengua catalana y que utilizan estos escolares bilingües cuando se expresan en la lengua castellana, como resultado de sus conocimientos de aquella lengua. Centrándonos en el campo de la producción y no en el de la percepción o comprensión, los fenómenos de interferencia tienen lugar en cada uno de los niveles lingüísticos: fónico, gramatical y léxico-semántico. En la necesidad de acotar el ámbito de análisis, hemos optado en esta ocasión por presentar los resultados obtenidos en el nivel fónico. Una vez obtenidos estos rasgos, los clasificaremos de acuerdo a una tipología lingüística (Weinreich, 1974; Payrató, 1985). Esperamos, por último, que su descripción nos permita mostrar qué factores estructurales favorecen la interferencia lingüística.

\section{Clasificación de los fenómenos fónicos}

\section{III.A. Interferencia fónica segmental}

\section{1) Subestimación de una distinción}

Proceso de simplificación por el cual se suprime una diferencia fónica entre dos sonidos del castellano, que se confunden en uno solo, por la influencia del catalán.

$-[0] /[\mathrm{u}]$ átonas $\rightarrow[\mathrm{u}]$

El catalán de Mallorca distingue los dos sonidos vocálicos [o] / [u] como el catalán occidental, pero el catalán de Sóller (Campos, Porreres) es una excepción al cerrar articulatoriamente la [o] hasta convertirla en [u], confudiéndose así la [o] y la [u] en el mismo grado que el catalán de Barcelona, coincidiendo con el menorquín y el ibicenco, pero discrepando de los otros pueblos de Mallorca en el tratamiento de la [o] átona. 
Por influencia del solleric (variante diatópica del mallorquín), los escolares cuando hablan castellano dejan de realizar la oposición funcional [o] / [u] al tender a cerrar las [o] átonas: Parecía un fide[u]. Pintarem[u]s las sillas verdes.

Como reflejo de la interferencia fónica encontramos también casos de confusión gráfica, como muchila en lugar de mochila.

$$
\text { _ [e] / [a] átonas } \rightarrow \text { [_] }
$$

El catalán de Mallorca, como el oriental, suprime la diferencia entre la [a] y la [e] y confunde estos sonidos en la vocal llamada e neutra o mixta [_]. De este modo, cuando los escolares que son bilingües en las dos lenguas hablan castellano dejan de realizar la oposición del castellano normativo de los no bilingües y dicen, por ejemlo: El z[_]p[_] tero no estaba. La [_]st[_]ción está detrás de la plaça.

$$
[\theta] /[s] \rightarrow[s]
$$

Deja de funcionar la oposición $[\theta] /$ [s] con la pérdida de la diferencia en el punto de articulación. Esta tendencia a pronunciar la fricativa interdental sorda $[\theta]$ como fricativa alveolar sorda [s] (seseo) en el discurso castellano de estos niños bilingües se produce por el influjo del catalán, que desconoce el sonido de la $[\theta]$ castellana.

La simple comparación de los sistemas fonológicos del catalán y del castellano explica muchos de los fenómenos de interferencia. Así, la presencia de ciertos fonemas propios sólo de uno de los dos sistemas puede llevar a fusiones o subestimaciones en la otra lengua, como ocurre en ente caso con la oposición castellana $[\theta] /[\mathrm{s}]$. El rechazo sistemático de la interdental [_] $]^{7}$, que apenas se encuentra en el habla de los niños bilingües, es favorecido por una serie de razones que aduce Payrató (1985: 100): El sonido [_] ha desaparecido en muchas variantes del castellano, algunas de las cuales se encuentra en contacto con variantes del catalán. En segundo lugar, el bajo rendimiento funcional de la oposición fonológica $[\theta$ ] / [s] en castellano favorece la interferencia, la fusión de los dos fonemas, «tant en l'aspecte productiu (castellà de molts catalans) com en el perceptiu; ambdós fenòmens contribueixen, de retruc, a l'adaptació de $[\theta]$ en $[s] »$.

La supresión de la diferencia en el punto de articulación de la pareja [ $\theta]$ / [s] queda reflejada en la pronunciación: La vaca tuvo un percan[s]e... y en la escritura, donde la interferencia gráfica es el reflejo de la interferencia fónica: Caperusita, colaborasió, produsió, resientes, la siguala (cast. cigarra), hase. En ocasiones el reflejo gráfico de la interferencia no es una s, sino la grafía ç, cuya pronunciación equivale a la [s] castellana, sobre todo si la palabra afectada existe como tal en catalán: Si los soldados abansan ocuparan la plaça fuerte.

7 Las explicaciones acerca del diferente comportamiento de los sonidos $[\mathrm{x}]$ y $[\theta]$ en catalán -progresión y rechazo- nos las proporcionan los artículos de Mariner (1953), Cerdà (1967), Mariner (1976) y Veny (1994). 
La líquida lateral palatal sonora desaparece por un proceso de deslateralización y se convierte en la fricativa central palatal sonora []. La pérdida de esta distinción (yeísmo) en el castellano hablado por estos escolares se ve favorecida por el yeísmo catalán del habla de Las Baleares ${ }^{8}$ —resultado [] en lugar de [ $\left.\lambda\right]$ (ll) y por factores gráficos. Mientras el mallorquín tiene una grafía $l l$ para el sonido lateral $[\lambda]$ equivalente a la grafía y al sonido castellano, la grafía $y$ castellana correspondiente a la fricativa [] central (mayo) es equivalente a las grafías catalanas $i$ de joia [__ ] y parecida a la grafía $j$ de neteja [n_t_a] o jo [_o] y a la grafía $g$ de pagès [p_s].

Las confusiones gráficas, las numerosas alternancias $l l / y / i / j / g$ en la escritura prueban esta interferencia fónica: estaba llo; cuando los pages del conde no siguieron al zorro; plailas, plallas; destrullen, ralloyos (rayos), disminulló, que valla a traerle, ollen ... .

\section{2) Sobrestimación de una distinción}

Formación de una diferencia fónica en la lengua receptora, donde no es necesaria, por imposición de las distinciones del sistema catalán.

$$
\text { - }[0] \rightarrow[0] /[\text { [_] }
$$

$$
[\mathrm{e}] \rightarrow[\mathrm{e}] /\left[{ }_{-}\right]
$$

Este proceso de diversificación puede ser inferido de la comparación de los sistemas fonológicos en contacto. Mientras que el sistema vocálico del castellano presenta tres grados de abertura: $[\mathrm{i}, \mathrm{u}] /[\mathrm{e}, \mathrm{o}] /[\mathrm{a}]$, el sistema vocálico catalán presenta cuatro grados de abertura: $[\mathrm{i}, \mathrm{u}] /[\mathrm{e}, \mathrm{o}] /[\ldots,-] /[\mathrm{a}]$.

La presencia de estas distinciones en catalán - [_], [_] — distorsiona el timbre de las vocales castellanas [e], [o], que se pronunciarán más abiertas en el habla de estos escolares: $d[$ [_] ber en lugar de $d[\mathrm{e}]$ ber por influencia del catalán deure [d_r_] $;$ Migu[_] $l$ por Migu[e]l, por influencia del catalán Miquel [mik_I]. En menor medida hemos encontrado ejemplos de [o] castellanas transformadas en [_]: [_] sio (ocio), [_]ca, (oca), $b$ [_] te (bote) por influencia de pot [p_t].

\section{3) Reinterpretación de una distinción}

Se transforma una diferencia fonológica en otra a través de una relación concreta que se establece entre los elementos de ambos sistemas.

Se trata de un fenómeno más complicado, pues supone un cambio en la relación entre los elementos del castellano por la influencia del catalán. No hemos encontrado ningún ejemplo que pueda ilustrar este proceso particular.

8 El habla de las Baleares, el de las Pitiusas y el de la zona oriental de Cataluña presentan yeísmo - resultado en lugar de $\lambda(l l)$ - (i semivocal en lugar de la l palatal) cuando la palatal proviene de los grupos latinos C'L, P'L, G'L, LI: abeia, reia, escui, fuia, ui en lugar de abella, rella, escull. fulla, ull. Cuando la $[\lambda]$ iba detrás de la vocal $i$, la î resultante quedó absorbida por la vocal y ha desaparecido de la pronunciación: conill $>$ conii $>$ coní; filla $>$ fiia $>$ fia. (Moll, F. de B., 1990 :68-69). 


\section{4) Sustitución}

Por un principio de economía el hablante bilingüe identifica sonidos que son parecidos en las dos lenguas, y sustituye uno por otro. Según Weinreich (1974) la distinta pronunciación de fonemas equivalentes es uno de los factores que estimulan la interferencia.

$-[\mathrm{d}]$ implosiva $\rightarrow[\mathrm{t}]$

En catalán toda consonante que no sea una líquida, en posición final se pronuncia sorda. En consecuencia, el alumno que es bilingüe en las dos lenguas encuentra gran dificultad en pronunciar la [d] final castellana (sonora) y la sustituye por su pareja sorda [t], con la que comparte el punto de articulación (dental). Esta interferencia fónica es muy común y encuentra su reflejo en la interferencia gráfica, identificándose grafía y sonido en ejemlos como: Le gusta la tempestat; la verdat es que... .

_[1] alveolar $\longrightarrow$ [1] velar

La resonancia velar de la [1] catalana no la tiene la castellana. Como se trata de dos sonidos parecidos, el alumno cuando habla castellano velariza la [1] castellana como si de una [1] catalana se tratara: Me hace ma[_].

[l1] $\rightarrow$ [11] geminada

Si se trata de palabras cuya correspondiente catalana tiene una 1.1 [11], ele geminada también velar, el hablante bilingüe pronunciará una ele geminada en castellano en lugar de una alveolar simple: co[1l]egio (cat. col.legi).

$$
[-1] \rightarrow[\lambda]
$$

La ele final de palabra es alveolar en castellano, pero palatal en catalán. Si esta lengua distingue $/ \lambda / / / 1 /$ en posición implosiva, el castellano neutraliza la oposición. El escolar identifica estos sonidos y realiza en ocasiones palatalizaciones de la -l implosiva cuando habla castellano: $e[\lambda]$ inspetor.

\section{$[\mathrm{x}] \rightarrow[\gamma]$}

En general, no hay ningún problema a la hora de pronunciar la $j[\mathrm{x}]$ castellana, y de hecho el catalán ha importado este sonido, que desconocía en su lengua, del castellano. Excepcionalmente hemos encontrado algún caso en que este sonido se adaptaba a otro del mismo orden velar, aunque sonoro, $[\gamma]$, por influencia de la palabra correspondiente catalana: dejar $>$ de $[\gamma] a r$ (cat. deixar, plegar), así como cierta tendencia leve a pronunciar la $\mathrm{j}[\mathrm{x}]$ como una oclusiva $[\mathrm{k}]$.

$$
-[\mathrm{t}] \mathrm{t} \rightarrow[\mathrm{J}] \text { (cat. } \mathrm{x})
$$

El sonido ch $[t]]$ del castellano se sustituye cuando no está en posición interna e intervocálica, que se conserva [t]], por su variante fricativa [ [], ya que teniendo en común el punto de articulación (palatales) y la ausencia de vibración de las cuerdas vocales (sordas), difieren en el modo de articulación (africada / fricativa). Con esta sustitución se confunden entonces la ch [t $[$ ] castellana con el sonido []] del catalán, que corresponde a las grafías catalanas $x$ o ix (xocolata, caixa): fe a buscar [J]ocolate blanco. 
$-{ }^{-X}-[\gamma s],[s] \rightarrow[j]$

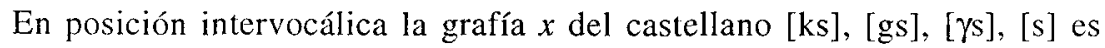
pronunciada como la grafía catalana $x$, que tiene un sonido parecido al de la $c h$ francesa [ [] chaise, más parecido a la [t]] castellana que a la [s] fricativa alveolar

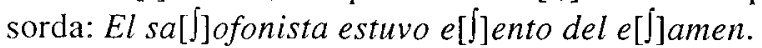

\section{5) Importación}

Se produce cuando el castellano recibe un elemento extraño que, sin embargo, no sirve para sustituir a otro propio como ocurre en el fenómeno de la sustitución.

- v [v]

En el castellano actual las grafías b y v corresponden ambas al mismo sonido bilabial [b]. En cambio, en Mallorca pronuncian la v como labiodental. De ahí que al hablar castellano se pronuncie la v como labiodental y no como bilabial: $\mathrm{La}[\mathrm{v}] \mathrm{aca} t \mathrm{tu}[\mathrm{v}]$ o. Este tipo de interferencia fónica produce numerosos ejemplos de interferencias gráficas: ivan, quitava, havia, govierno, y también casos de ultracorrecciones: gabiotas, ibernadero (gaviotas, invernadero).

\section{6) Pérdida}

Ese fenómeno, que se da cuando el castellano como lengua objeto de la interferencia deja de utilizar una determinada diferencia fónica o un elemento cualquiera que le es propio por el influjo de la lengua catalana, donde no se da tal oposición o donde no existe tal elemento, es aplicable a los casos de desaparición del primer apartado (subestimación de una distinción), desde el punto de vista de la lengua receptora.

En la combinación de unidades se dan fenómenos paralelos:

-Asimilación: Sonorización de la -s final de palabra en contacto con vocal.

En catalán toda sibilante final de vocablo en contacto con la inicial vocálica de otro, es sonora. En consecuencia, los niños que son bilingües en las dos lenguas, al hablar castellano pronuncian: $l o[z]$ animales, con s sonora en lugar de la pronunciación castellana con [s] s sorda.

-Africación: El mallorquín y el menorquín pronuncian africado el sonido resultante del contacto de una [s] implosiva y otra explosiva cuando hablan castellano por influencia de sus lenguas. De este modo, nos encontramos en el habla de los chicos encuestados con una africada [ts] en lugar de una [s:] s larga, resultado de la suma de estas dos [s], como encontramos en el discurso castellano de los no bilingües: la[ts]emanas, la[ts]illas verdes.

-Sustitución: Se da también en la diptongación. Así como en catalán no existe el diptongo ia [ja] y en castellano sí, los escolares tienden a pronunciar la $i$ como vocal y no como semiconsonante: Estud[i]amos mucho.

-Ultracorrección: Por analogía con treinta pronuncian cuar[ei]nta, cincu[ei]nta. Como al trenta catalán corresponde treinta en castellano, al quaranta y al cinquanta deberían corresponder en castellano cuareinta y cincueinta. 


\section{III.B Interferencia fónica suprasegmental}

En el campo de la acentuación destaca el traslado de acento, que se ve favorecido por la semejanza formal entre los elementos de ambas lenguas y la consiguiente identificación interlingüística de las unidades. Así, nos encontramos con los siguientes ejemplos:

- acentuación aguda de pero [peró] por influencia del correspondiente catalán peró [peró]; acentuación aguda de sino [sinó] por influjo del catalán sinó [sinó].

- acentuación llana de la interrogación ¿por qué?, pronunciada [pórke] por haber sufrido el mallorquín un desplazamiento del acento de perquè en [pérke].

- la transferencia de acento es corriente también en formas verbales: estudia, habia, cambia, en los nombres propios: Rámon y en algunos comunes como: poésia.

En cuanto a la entonación creemos con Payrató (1985:111) que los bilingües utilizan normalmente un único modelo de entonación aplicado a las dos lenguas: «Repassant els estudis de Navarro Tomás i de Quilis en castellà, hom té la impressió que les corbes melòdiques del cat. i cast. són iguals pel que fa a les juntures terminals, i que les diferéncies provindrien especialment del contorn tonal de la frase i del binarisme accentual dels mots catalans».

\section{III.C. Interferencias gráficas}

En el lenguaje escrito son muchos los casos en que se alternan las dos lenguas, catalán y castellano: palabras similares aparecen en el discurso castellano de estos escolares, como setmana en lugar de semana; joventut en lugar de juventud (cat. joventut). En este caso, se trataría de una ultracorrección porque como los hablantes de Sóller cierran la [o] en [u], cuando oyen [u] creen que la grafía adecuada al castellano puede ser $o$.

Palabras catalanas sustituyen a las castellanas: cami por camino; bé en lugar de bien; també en lugar de también; escriptores por escritores; palabras que no he enten sustituyendo a la forma castellana he entendido por influencia del catalán he entès. Son múltiples las confusiones a la hora de representar el sonido de la nasal palatal entre las grafías castellana y catalana: senñora en lugar de señora (cat. senyora); montanya por montaña (cat. muntanya); las alternacias en las grafías de la conjunción copulativa $i / y: i$ hai, $i$ aquí, a dormir $i$ a comer y los cambios en la colocación de la tilde: és (cat.és); estó que dices (cat. això que dius).

\section{Conclusiones}

El castellano de las Islas Baleares se puede ver desde una perspectiva de lenguas en contacto. Esto se puede apreciar a nivel del individuo que utiliza los 
dos idiomas para fines comunicativos, y a nivel de sociedad donde de una manera diglósica existe la tendencia a emplear un idioma u otro dentro de esferas sociolingüísticas (casa, vecindad, trabajo, administración, medios de comunicación) y donde el castellano sigue estando asociado a los grupos de prestigio socio-político-cultural". El contacto entre el catalán y el castellano es más que un fenómeno lingüístico, porque detrás de los dos idiomas se encuentran relaciones interculturales que reflejan un proceso de coexistencia entre las dos comunidades lingüísticas.

De las muestras de uso real recogidas a lo largo del trabajo de campo que hemos llevado a cabo con la comunidad escolar del colegio público Es Puig de Sóller (Baleares) hemos obtenido una serie de rasgos fonéticos, que nos hablan de una influencia notable de los hábitos del catalán sobre el castellano de esta comunidad lingüística.

Estos rasgos lingüísticos se alejan de la norma culta del castellano de otras áreas geográficas; son provocados por el bilingüismo ambiental que existe en la comunidad balear y presentan una vitalidad real estimulados por la progresiva normalización social de la lengua catalana, que alcanza a la aceptación de muchos de ellos por la comunidad balear no bilingüe.

Estos rasgos peculiares, que como advirtiera Weinreich (1953/1974), no constituyen préstamos efímeros de una lengua a otra, sino fenómenos sistemáticos que ocurren en el habla de las comunidades bilingües no pueden ser vistos sólo como desviaciones de una organización gramatical básica, ya que ellos constituyen la propia gramática de esas comunidades (Romaine, 1988: 286).

Se caracteriza, por lo tanto, la gramática de uso en lengua castellana de esta comunidad bilingüe por los siguientes rasgos fonéticos: -Pérdida de la [o] átona, que pasa a $[u]$. -Pérdida de la oposición funcional a / e què se confunden en la e neutra. -El seseo: adaptación de la $[\theta]$, fricativa interdental sorda en [s], fricativa alveolar sorda. -El yeísmo: deslateralización de la $[\lambda]$, lateral palatal sonora que se convierte en la [], fricativa central palatal sonora. -Distorsión de las vocales $[\mathrm{e}],[\mathrm{o}]$, que se pronunciarán más abiertas. -Sustitución de sonidos: ensordecimiento de la dental ([d] > [t]); velarización de la [1]; geminación de la [1]; palatalización de la [-1] implosiva final de palabra; sonorización de la fricativa velar sorda $(\mathrm{j}[\mathrm{x}]>[\gamma])$; fricación de la ch $[\mathrm{t}[]$ que pasa a [ [] ; pronunciación de la-x-como [j]. -Importación de sonidos: la [v] labiodental. -Sonorización de la -s final de palabra en contacto con vocal. -Pronunciación africada del resultado de dos eses en contacto. -Vocalización de la i [j] semiconsonante, elemento integrante de un diptongo. -Ultracorrecciones. -Traslado de acento. Confusiones y alternancias gráficas entre las dos lenguas.

- Estaríamos en una fase de bilingüisno dinámico, en la que el catalán amenaza con desplazar, al castellano, ya que las dos lenguas comienzan a usarse, indistintamente, para varias funciones reservadas antes a una de las lenguas. 
Estos rasgos se desarrollan por la actuación de diversos factores estructurales y no estructurales; entre los primeros cabe señalar la economía, generalización de los elementos comunes y supresión de los más específicos; la frecuencia en el discurso de las unidades afectadas; y la inteligibilidad, la posibilidad de hacerse entender. Entre los no estructurales (mostrados en el Cuestionario) destacamos la habilidad linguística de los escolares, la especialización en el uso de cada lengua por temas e interlocutores, la homogeneidad del grupo bilingüe, las diferencias de poder y prestigio en la consideración de las dos lenguas, y la tolerancia con respecto a la mezcla de lenguas y a los usos no normativos de cada una de ellas.

\section{Referencias bibliográficas:}

APPEL, R. y P. MUYSKEN, (1987): Language contact and bilingualism, London, Edward Arnold.

ARACIL, LL. V. (1973): «Bilingualism as a Myth», Interamerican Review, 24, pp. 521-533.

BAETENS, H. (1986): Bilingualism: basic principles, Clevedon, Multilingual Matters Ltd.

BIBILONI, G. (1988): «Universitat, llengua i societat a les Illes Balears», Caplletra, 3, Revista de Filologia, 1988, pp. 65-75.

CERDÁ, R. (1967): «Apreciaciones generales sobre cast. $/ x / \longrightarrow$ cat. [x] en el Campo de Tarragona, Revista de Filología Española, L, 57-96.

DAOUST, D. y J. MAURAIS (1987): «L'aménagement linguistique», en MAURAIS, J.(ed.): Politique et Aménagement linguistiques, Québec, Conseil de la langue française, Paris, Le Robert, pp. 5-46.

ETXEBARRÍA, M. (1986): «El castellano actual en el País Vasco: Estudio de interferencias» en AA.VV., : El castellano actual en las comunidades bilingïes de España, Salamanca, Junta de Castilla y León, pp. 65-91.

FERGUSON, C. A. Y J. J. GUMPERZ (1959): «Diglossia», Word, 15, pp. 325340.

GARDNER-CHLOROS, P. (1983): «Code-switching: approches principales et perspectives», La Linguistique, 19, 1983, 2, Paris, pp. 21-53.

HALLIDAY, M. A. K.; McINTOSCH, A.; STREVENS, P. (1968): «The Users and Uses of Language», en FISHMAN, J. A. (ed.): Readings in the Sociology of Language, The Hague/Paris, Mouton, pp. 139-169.

HAUGEN, E. (1953): The Norwegian Languaje in America, Philadelphia, Univ. of Pennsylvania Press.

HAUGEN, E. (1973): «Bilingualism, Language Contact and Inmigrant Languages in the United States. A Research Report 1956-1970», en SEBEOK, Th. A. (Ed.): Current Trends in Linguistics, 10, 1, The Hague/Paris, Mouton, pp. 505-591. 
JORDANA, C. A. (1968): El catalá y el castellá comparats, Barcelona, Ed. Barcino.

LEWIS, G. (1972): Multilingualism in the Soviet Union, The Hague, Mouton. MACKEY, W. F. (1976): Bilinguisme et contact des langues, Paris, Klincksieck. MARINER, S. (1953): «Castellanismos léxicos en un habla local del Campo de Tarragona», Boletín de la Real Academia de Buenas Letras de Barcelona. $X X V, I, J 7 J-226$.

MARINER, S. (1976): «El préstamo fonológico», Revista Española de Lingüística, 6, 2, 301-308.

MOLLÀ , T. y C. PALANCA (1987): Curs de Sociolingüística I, Alzira, Edicions Bromera.

MOLL, F. de B. (1990): El parlar de Mallorca, Palma de Mallorca, Editorial Mol.

ROJO, G. (1981): "Conductas y actitudes lingüísticas en Galicia», Revista Española de Lingüística, 11, 2, pp. 269-298.

PAYRATÓ, Ll. (1985): La interferència lingüística (Comentaris i exemples català-castellà), Barcelona, Curial-Publicacions de l'Abadia de Montserrat. ROMAINE, S. (1988): Pidgin and Creole Languages, Longman, Harlow.

ROTAETXE, K. (1988): Sociolingüística, Madrid, Síntesis.

SÁNCHEZ CARRIÓN, J. M. (1981): «La Navarra cantábrica», Fontes Linguae Vasconum, 37a y b, Pamplona, pp. 1-26 y 27-78.

SANDFELD, K. (1936/1938): «Problèmes d'interférences linguistiques», Actes du Quatrième Congrès International des Linguistes, Copenhaguen, pp. 59-61. SECO, M. (1977): «El léxico de hoy», Comunicación y lenguaje, Madrid, Karpos, pp. 183-201.

SERRANO, M. C. (1990): «Análisis del comportamiento lingüístico en una situación bilingüe», en Estudis Balearics, 36, Palma de Mallorca, Institut d'Estudis Balearics-C.S.I.C., pp. 63-82.

SIGUAN, M. (1976): «Bilingüismo y Sociología», Revista Española de Lingüistica, 6.1., Madrid, pp. 27-88.

SIGUAN, M. y W. E. MACKEY (1986): Educación y bilingiïismo, Madrid, Santillana-UNESCO.

TABOURET-KELLER, A. (1982): «Entre Bilinguisme et Diglosssie. Du malaise des cloisonnements universitaires au malaise social», La Linguistique, 18, 1982, 1, Paris, pp. 17-43.

VENY, J. (1994): «La importación del fonema/x/ en catalán», Revista Española de Lingüística, 24, 1, Madrid, pp. 1-19.

VAN OVERBEKE, M. (1976): Mécanismes de l'interférence linguistique, Madrid, Fragua.

WEINREICH, U. (1953/1974): Languages in contac, The Hague, Mouton. Lenguas en contacto. Descubrimientos y problemas, Caracas, Ediciones de la Biblioteca Central de Venezuela. 